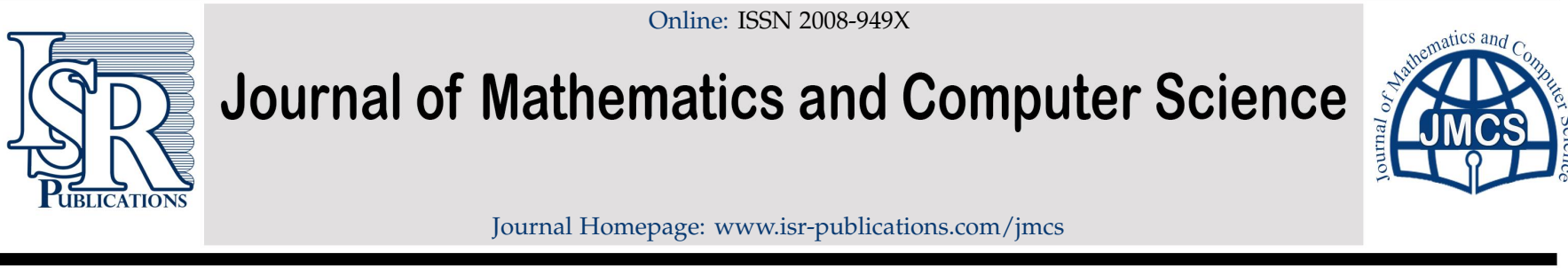

\title{
Gronwall inequality and existence of solutions for differen- tial equations with generalized Hattaf fractional derivative
} Check for updates

\author{
Khalid Hattaf ${ }^{a, b, *}$, Ahmed A. Mohsen ${ }^{c, d}$, Hassan F. Al-Husseiny ${ }^{\mathrm{e}}$ \\ ${ }^{a}$ Equipe de Recherche en Modélisation et Enseignement des Mathématiques (ERMEM), Centre Régional des Métiers de l'Education et \\ de la Formation (CRMEF), 20340 Derb Ghalef, Casablanca, Morocco. \\ ${ }^{b}$ Laboratory of Analysis, Modeling and Simulation (LAMS), Faculty of Sciences Ben M'sik, Hassan II University of Casablanca, P.O. Box \\ 7955, Sidi Othman, Casablanca, Morocco. \\ ${ }^{c}$ Department of Mathematics, College of Education for Pure Science (Ibn Al-Haitham), University of Baghdad, Iraq. \\ ${ }^{d}$ Ministry of Education, Rusafa 1, Baghdad, Iraq. \\ ${ }^{e}$ Department of Mathematics, College of Science, University of Baghdad, Iraq.
}

\begin{abstract}
The classical Gronwall inequality is one of the basic tools in the theory of differential and integral equations. In this paper, a new version of this inequality is presented and extended to differential equations with the generalized Hattaf fractional derivative involving non-singular kernel. The existence and uniqueness of solutions for such last type of fractional differential equations are rigorously investigated. Furthermore, an application is presented to study the Ulam-Hyers stability of certain equations.
\end{abstract}

Keywords: Gronwall inequality, Hattaf fractional derivative, fractional differential equation, Ulam-Hyers stability.

2020 MSC: 26A33, 26D15, 34D10, 34D20.

(C)2022 All rights reserved.

\section{Introduction}

The first version of Gronwall inequality was introduced by Gronwall [16] in 1919. Since this date, several continuous and discrete versions of this inequality have been established in order to study the quantitative and qualitative properties of differential equations as well as difference equations. For instance, the generalizations of the classical Gronwall inequality in the frame of integral equation with singular kernel have been presented by Dixon and McKee [12]. Recently, Adjabi et al. [1] obtained a Gronwall inequality for generalized fractional operators unifying Riemann-Liouville and Hadamard fractional operators. In 2018, Jarad et al. [19] established a Gronwall inequality in the frame of Atangana-Baleanu fractional integral. In 2019, Alzabut et al. [6] provided a new version for the Gronwall inequality in the frame of the generalized proportional fractional derivative.

\footnotetext{
*Corresponding author

Email address: k. hattaf@yahoo.fr (Khalid Hattaf)

doi: $10.22436 /$ jmcs.027.01.02
}

Received: 2021-11-24 Revised: 2021-12-28 Accepted: 2022-01-07 
In 2020, a new generalized definition of fractional derivative with non-singular kernel for both Caputo and Riemann-Liouville types was introduced in [17] in order to investigate the impact of memory effect on the dynamics of some dynamical systems in epidemiology and virology. Such definition includes the most famous fractional derivatives with non-singular kernels existing in the literature such as the Caputo-Fabrizio fractional derivative [10], the Atangana-Baleanu fractional derivative [7], and the weighted Atangana-Baleanu fractional derivative [4].

The main purpose of this paper is to derive a new version of Gronwall inequality as well as to prove the existence and uniqueness of solutions for differential equations with the new generalized Hattaf fractional (GHF) derivative [17]. Recently, this new derivative was used to describe the dynamics of Cholera transmission [11]. Furthermore, the advantages of using the GHF operator are the non-locality and nonsingularity of its kernel described by the Mittag-Leffler function with a parameter and exponent different from the order of the fractional derivative, unlike the Atangana-Baleanu type of fractional derivative which has a kernel whose parameter and exponent coincide with the order of the derivative. Also, the GHF operator covers the fractional derivatives with non-singular kernels intrduced in [4, 7, 10], and it contains a weight function which can be used to write and solve several integral equations in an elegant way as presented in $[2,5,9]$. In addition, the GHF derivative can be applied to real-world problems as in [21-23] and $[3,14,15,20]$. On the other hand, the contributions of the present paper are the extension of the Gronwall inequality to fractional differential equations (FDEs) involving the GHF derivative, the discussion of the existence, the uniqueness as well as the Ulam-Hyers stability conditions of such FDEs, and also the generalization of the results related to a class of ordinary differential equations with Atangana-Baleanu fractional derivative investigated in [19].

The structure of the rest of this paper is organized as follows. The next section is devoted to the preliminaries including definitions and some fundamental properties of the GHF derivative with nonsingular kernel in Caputo sense, which will be used in the subsequent sections. Section 3 establishes the Gronwall inequality within the GHF derivative. Section 4 deals with the existence and uniqueness of solutions. An application of our main results is presented in Section 5. In the last section, we give a conclusion and prospects for our future work.

\section{Preliminaries}

This section recalls some important results and definitions related to the GHF derivative with nonsingular kernel that will be necessary in the sequel.

Definition 2.1. Let $\alpha \in[0,1), \beta, \gamma>0$, and $f \in H^{1}(a, b)$. The GHF derivative of order $\alpha$ in Caputo sense of the function $f(t)$ with respect to the weight function $w(t)$ is defined as follows [17],

$$
{ }^{C} D_{a, t, w}^{\alpha, \beta, \gamma} f(t)=\frac{N(\alpha)}{1-\alpha} \frac{1}{w(t)} \int_{a}^{t} E_{\beta}\left[-\mu_{\alpha}(t-\tau)^{\gamma}\right] \frac{d}{d \tau}(w f)(\tau) d \tau,
$$

where $w \in C^{1}(a, b), w, w^{\prime}>0$ on $[a, b], N(\alpha)$ is a normalization function obeying $\mathrm{N}(0)=\mathrm{N}(1)=1$, $\mu_{\alpha}=\frac{\alpha}{1-\alpha}$ and $E_{\beta}(t)=\sum_{k=0}^{+\infty} \frac{t^{k}}{\Gamma(\beta k+1)}$ is the Mittag-Leffler function of parameter $\beta$.

In the above definition, $\mathrm{H}^{1}(\mathrm{a}, \mathrm{b})$ is the Sobolev space of order one defined as follows

$$
\mathrm{H}^{1}(\mathrm{a}, \mathrm{b})=\left\{\mathrm{u} \in \mathrm{L}^{2}(\mathrm{a}, \mathrm{b}): \mathrm{u}^{\prime} \in \mathrm{L}^{2}(\mathrm{a}, \mathrm{b})\right\} .
$$

Furthermore, the GHF derivative presented in this definition generalizes and includes various cases available in the literature. For example, when $w(t)=1$ and $\beta=\gamma=1,(2.1)$ reduced to the Caputo-Fabrizio fractional derivative [10] given by

$$
{ }^{C} D_{a, t, 1}^{\alpha, 1,1} f(t)=\frac{N(\alpha)}{1-\alpha} \int_{a}^{t} \exp \left[-\mu_{\alpha}(t-\tau)\right] f^{\prime}(\tau) d \tau .
$$


When $w(t)=1$ and $\beta=\gamma=\alpha$, (2.1) reduced to the Atangana-Baleanu fractional derivative [7] given by

$$
{ }^{C} D_{a, t, 1}^{\alpha, \alpha, \alpha} f(t)=\frac{N(\alpha)}{1-\alpha} \int_{a}^{t} E_{\alpha}\left[-\mu_{\alpha}(t-\tau)^{\alpha}\right] f^{\prime}(\tau) d \tau
$$

In addition, the weighted Atangana-Baleanu fractional derivative [4] defined as follows

$$
{ }^{C} D_{a, t, w}^{\alpha, \alpha} f(t)=\frac{N(\alpha)}{1-\alpha} \frac{1}{w(t)} \int_{a}^{t} E_{\alpha}\left[-\mu_{\alpha}(t-\tau)^{\alpha}\right] \frac{d}{d \tau}(w f)(\tau) d \tau
$$

is a particular case of GHF derivative when $\beta=\gamma=\alpha$.

For simplicity, denote ${ }^{C} D_{a, t, w}^{\alpha, \beta, \beta}$ by $\mathcal{D}_{a, w}^{\alpha, \beta}$. It follows from [17] that the generalized fractional integral associated to $\mathcal{D}_{\mathrm{a}, \mathrm{w}}^{\alpha, \beta}$ is given by the following definition.

Definition 2.2 ([17]). The generalized fractional integral operator associated to $\mathcal{D}_{a, w}^{\alpha, \beta}$ is defined by

$$
\mathcal{J}_{a, w}^{\alpha, \beta} f(t)=\frac{1-\alpha}{N(\alpha)} f(t)+\frac{\alpha}{N(\alpha)} R_{\mathcal{J}_{a, w}}^{\beta} f(t),
$$

where ${ }^{R L} \mathcal{J}_{\mathfrak{a}, w}^{\beta}$ is the standard weighted Riemann-Liouville fractional integral of order $\beta$ defined by

$$
\operatorname{RL}_{\mathcal{J}_{a, w}} \mathrm{f}(\mathrm{t})=\frac{1}{\Gamma(\beta)} \frac{1}{\mathcal{w}(\mathrm{t})} \int_{\mathbf{a}}^{\mathrm{t}}(\mathrm{t}-\tau)^{\beta-1} \mathcal{w}(\tau) \mathrm{f}(\tau) \mathrm{d} x
$$

Remark 2.3. If we take $w(t)=1$ and $\beta=\alpha$ in (2.2), then the Atangana-Baleanu fractional integral operator [7] is obtained.

The following result recall the fundamental theorem that extends the Newton-Leibniz formula presented in $[8,13]$.

Theorem 2.4 ([18]). Let $\alpha \in[0,1), \beta>0$ and $\mathrm{f} \in \mathrm{H}^{1}(\mathrm{a}, \mathrm{b})$. Then we have the following properties:

$$
\mathcal{J}_{a, w}^{\alpha, \beta}\left(\mathcal{D}_{a, w}^{\alpha, \beta} f\right)(t)=f(t)-\frac{w(a) f(a)}{w(t)},
$$

and

$$
\mathcal{D}_{a, w}^{\alpha, \beta}\left(\mathcal{J}_{a, w}^{\alpha, \beta} f\right)(t)=f(t)-\frac{w(a) f(a)}{w(t)}
$$

\section{Gronwall inequality via GHF derivative}

In this section, we establish a Gronwall inequality in the frame of fractional integrals associated with the GHF derivative. First, we need the following lemma.

Lemma 3.1. Let $\beta>0, x(t), u(t)$ be nonnegative functions on $[a, b)$ and $v(t)$ be nonnegative and nondecreasing function on $[\mathrm{a}, \mathrm{b})$ such that $v(\mathrm{t}) \leqslant \mathrm{M}$, where $M$ is a constant. If

$$
x(t) \leqslant u(t)+v(t)^{R L_{\mathcal{J}} \beta, w}{ }^{\beta} x(t),
$$

then

$$
x(t) \leqslant u(t)+\int_{a}^{t} \sum_{n=1}^{+\infty} \frac{(v(t))^{n}}{\Gamma(n \beta)}(t-\tau)^{n \beta-1} u(\tau) d \tau, \quad t \in[a, b) .
$$


Proof. The proof of this lemma is based on the result presented in [1, Theorem 2.1].

From (2.3) and (3.1), we have

$$
w(t) x(t) \leqslant w(t) u(t)+\frac{v(t)}{\Gamma(\beta)} \int_{a}^{t}(t-\tau)^{\beta-1} w(\tau) x(\tau) d \tau
$$

Since the three functions $w(t) x(t), w(t) u(t)$ and $\frac{v(t)}{\Gamma(\beta)}$ satisfied the hypotheses of [1, Theorem 2.1], we deduce that

$$
\begin{aligned}
w(t) x(t) & \leqslant w(t) u(t)+\int_{a}^{t} \sum_{n=1}^{+\infty} \frac{(v(t))^{n}}{\Gamma(n \beta)}(t-\tau)^{n \beta-1} w(\tau) u(\tau) d \tau \\
& \leqslant w(t) u(t)+w(t) \int_{a}^{t} \sum_{n=1}^{+\infty} \frac{(v(t))^{n}}{\Gamma(n \beta)}(t-\tau)^{n \beta-1} u(\tau) d \tau
\end{aligned}
$$

Hence,

$$
x(t) \leqslant u(t)+\int_{a}^{t} \sum_{n=1}^{+\infty} \frac{(v(t))^{n}}{\Gamma(n \beta)}(t-\tau)^{n \beta-1} u(\tau) d \tau
$$

This completes the proof.

Theorem 3.2. Let $\beta>0, x(t), u(t)$ be nonnegative functions on $[a, b)$ and $v(t)$ be nonnegative and nondecreasing function on $[a, b)$ such that $v(t) \leqslant M$, where $M$ is a constant satisfying $N(\alpha)-(1-\alpha) M>0$. If

$$
x(t) \leqslant u(t)+v(t) \mathcal{J}_{a, w}^{\alpha, \beta} x(t),
$$

then

$$
x(t) \leqslant \frac{N(\alpha) u(t)}{N(\alpha)-(1-\alpha) v(t)}+\int_{a}^{t} \sum_{n=1}^{+\infty} \frac{N(\alpha)(\alpha v(t))^{n}(t-\tau)^{n \beta-1} u(\tau)}{\Gamma(n \beta)[N(\alpha)-(1-\alpha) v(t)]^{n}[N(\alpha)-(1-\alpha) v(\tau)]} d \tau
$$

Proof. According to (2.2) and (3.2), we get

$$
x(t) \leqslant \frac{N(\alpha) u(t)}{N(\alpha)-(1-\alpha) v(t)}+\frac{\alpha v(t)}{N(\alpha)-(1-\alpha) v(t)} R_{\mathcal{J}} \mathcal{J}_{a, w} x(t)
$$

Let $U(t)=\frac{N(\alpha) u(t)}{N(\alpha)-(1-\alpha) v(t)}$ and $V(t)=\frac{\alpha v(t)}{N(\alpha)-(1-\alpha) v(t)}$. It is not hard to see that the three functions $x(t)$, $\mathrm{U}(\mathrm{t})$ and $\mathrm{V}(\mathrm{t})$ satisfy the hypotheses of Lemma 3.1. Thus,

$$
x(t) \leqslant u(t)+\int_{a}^{t} \sum_{n=1}^{+\infty} \frac{(v(t))^{n}}{\Gamma(n \beta)}(t-\tau)^{n \beta-1} u(\tau) d \tau .
$$

This implies (3.3).

Corollary 3.3. Under the hypotheses of Theorem 3.2, assume further that $u(t)$ is a nondecreasing function on $[a, b)$. Then

$$
x(t) \leqslant \frac{N(\alpha) u(t)}{N(\alpha)-(1-\alpha) v(t)} E_{\beta}\left(\frac{\alpha v(t)(t-a)^{\beta}}{N(\alpha)-(1-\alpha) v(t)}\right) .
$$

Proof. According to (3.3) and the assumption that $u(t)$ is a nondecreasing function on $[a, b)$, we have

$$
x(t) \leqslant \frac{N(\alpha) u(t)}{N(\alpha)-(1-\alpha) v(t)}\left[1+\int_{a}^{t} \sum_{n=1}^{+\infty} \frac{(\alpha v(t))^{n}(t-\tau)^{n \beta-1}}{\Gamma(n \beta)[N(\alpha)-(1-\alpha) v(t)]^{n}} d \tau\right]
$$


Then

$$
\begin{aligned}
x(t) & \leqslant \frac{N(\alpha) u(t)}{N(\alpha)-(1-\alpha) v(t)}\left[1+\sum_{n=1}^{+\infty} \frac{(\alpha v(t))^{n}}{\Gamma(n \beta)[N(\alpha)-(1-\alpha) v(t)]^{n}} \int_{a}^{t}(t-\tau)^{n \beta-1} d \tau\right] \\
& =\frac{N(\alpha) u(t)}{N(\alpha)-(1-\alpha) v(t)}\left[1+\sum_{n=1}^{+\infty} \frac{(\alpha v(t))^{n}(t-a)^{n \beta}}{\Gamma(n \beta+1)[N(\alpha)-(1-\alpha) v(t)]^{n}}\right] \\
& =\frac{N(\alpha) u(t)}{N(\alpha)-(1-\alpha) v(t)} E_{\beta}\left(\frac{\alpha v(t)(t-a)^{\beta}}{N(\alpha)-(1-\alpha) v(t)}\right) .
\end{aligned}
$$

This completes the proof.

Remark 3.4. If we take $w(t)=1$ and $\beta=\alpha$ in Corollary 3.3, then Gronwall's inequality for AtanganaBaleanu fractional integrals in [19] is recovered. Also, the proofs of the above results are similar to ones in [6] for a conformable derivative, and in [1] for a fractional derivative with singular kernel.

For $v(t)=M \geqslant 0$ in Theorem 3.2 and Corollary 3.3, we have the following result.

Corollary 3.5. Let $\beta>0, x(t), u(t)$ be nonnegative functions and $v(t)=M \geqslant 0$ with $N(\alpha)-(1-\alpha) M>0$. If

$$
x(t) \leqslant u(t)+M_{a, w}^{\alpha, \beta} x(t)
$$

then

$$
x(t) \leqslant \frac{N(\alpha)}{N(\alpha)-(1-\alpha) M}\left[u(t)+\int_{a}^{t} \sum_{n=1}^{+\infty} \frac{(\alpha M)^{n}(t-\tau)^{n \beta-1} u(\tau)}{\Gamma(n \beta)[N(\alpha)-(1-\alpha) M]^{n}} d \tau\right] .
$$

Furthermore, if in addition $\mathrm{u}(\mathrm{t})$ is a nondecreasing function on $[\mathrm{a}, \mathrm{b})$, we have

$$
x(t) \leqslant \frac{N(\alpha) u(t)}{N(\alpha)-(1-\alpha) M} E_{\beta}\left(\frac{\alpha M(t-a)^{\beta}}{N(\alpha)-(1-\alpha) M}\right) .
$$

\section{Existence and uniqueness of solutions}

In this section, we study the existence and uniqueness of the following Cauchy problem

$$
\left\{\begin{array}{l}
\mathcal{D}_{a, w}^{\alpha, \beta} x(t)=f(t, x(t)) \\
x(a)=x_{0}
\end{array}\right.
$$

where $x_{0} \in \mathbb{R}$ and $f:[a, b] \times \mathbb{R} \rightarrow \mathbb{R}$ is continuous with $f(a, x(a))=0$.

Lemma 4.1. $x(t)$ is a solution of (4.1) if and only if $x(t)$ satisfies the following integral equation

$$
x(t)=\frac{w(a) x_{0}}{w(t)}+\mathcal{J}_{a, w}^{\alpha, \beta} f(t, x(t))
$$

Proof. Let $x(t)$ be a solution of (4.1). Applying the Hattaf fractional integral to both sides of (4.1) and using (2.4), we obtain

$$
x(t)=\frac{w(a) x(a)}{w(t)}+\mathcal{J}_{a, w}^{\alpha, \beta} f(t, x(t)) .
$$

Since $x(a)=x_{0}$, we have (4.2). Suppose now that $x(t)$ satisfies (4.2). Then

$$
\chi(a)=\frac{w(a) x_{0}}{w(a)}+\mathcal{J}_{a, w}^{\alpha, \beta} f(a, x(a))=x_{0}+\mathcal{J}_{a, w}^{\alpha, \beta} f(a, x(a)) .
$$


Since $f(a, x(a))=0$, we get $x(a)=x_{0}$. As $x(t)$ satisfies (4.2), we have

$$
\begin{aligned}
\mathcal{D}_{a, w}^{\alpha, \beta} x(t) & =\mathcal{D}_{a, w}^{\alpha, \beta}\left(\frac{w(a) x_{0}}{w(t)}\right)+f(t, x(t)) \\
& =0+f(t, x(t))
\end{aligned}
$$

Thus, $x(t)$ satisfies (4.1).

Throughout the rest part of this paper, let us assume that $f$ is Lipschitz in its second variable, i.e., it satisfies the following assumption:

$\left(\mathcal{A}_{1}\right)$ There exists a constant $\mathrm{L}>0$ such that

$$
\left|f\left(t, x_{1}\right)-f\left(t, x_{2}\right)\right| \leqslant L\left|x_{1}-x_{2}\right|, \quad \forall t \in[a, b], \text { and } x_{1}, x_{2} \in \mathbb{R} .
$$

Theorem 4.2. Assume that $\left(\mathcal{A}_{1}\right)$ holds and $\mathrm{L}<\frac{\mathrm{N}(\alpha)}{1-\alpha}$. If $\mathrm{x}$ and $\mathrm{y}$ are two solutions of $(4.1)$, then $\mathrm{x}=\mathrm{y}$. This implies the uniqueness of solutions.

Proof. Let $x$ and $y$ are two solutions of (4.1). According to Lemma 4.1, we have

$$
y(t)-x(t)=\mathcal{J}_{a, w}^{\alpha, \beta}(f(t, y(t))-f(t, x(t))) .
$$

Since the assumption $\left(\mathcal{A}_{1}\right)$ holds, we deduce that

$$
\left.|y(t)-x(t)| \leqslant L \mathcal{J}_{a, w}^{\alpha, \beta} \mid y(t)\right)-x(t) \mid
$$

Applying the Gronwall inequality in Corollary 3.5 to the above inequality, we obtain

$$
|y(t)-x(t)| \leqslant \frac{N(\alpha) \times 0}{N(\alpha)-(1-\alpha) L} E_{\beta}\left(\frac{\alpha L(t-a)^{\beta}}{N(\alpha)-(1-\alpha) L}\right)=0
$$

This implies $x(t)=y(t)$ for all $t \in[a, b]$.

Theorem 4.3. Assume that $\left(\mathcal{A}_{1}\right)$ holds. If $\mathrm{L}\left(\frac{1-\alpha}{\mathrm{N}(\alpha)}+\frac{\alpha(\mathrm{b}-\mathrm{a})^{\beta}}{\mathrm{N}(\alpha) \Gamma(\beta+1)}\right)<1$, then the Cauchy problem (4.1) has a unique solution.

Proof. Let $\mathcal{C}=\mathrm{C}([\mathrm{a}, \mathrm{b}], \mathbb{R})$ be the Banach space of continuous functions $\mathrm{g}$ from $[\mathrm{a}, \mathrm{b}]$ into $\mathbb{R}$ with the norm

$$
\|g\|_{\mathcal{e}}=\sup _{t \in[a, b]}|g(t)|
$$

Consider the operator $\Upsilon: \mathcal{C} \rightarrow \mathcal{C}$ as follows

$$
(\curlyvee x)(t)=\frac{w(a) x(a)}{w(t)}+\mathcal{J}_{a, w}^{\alpha, \beta} f(t, x(t)), \quad t \in[a, b] .
$$

From Lemma 4.1, it suffices to prove that the operator $\checkmark$ has a unique fixed point. As in [22], we first prove that $\checkmark$ is well defined. By (4.3), we have

$$
\begin{aligned}
|(\Upsilon x)(t)| & =\left|\frac{w(a) x(a)}{w(t)}+\mathcal{J}_{a, w}^{\alpha, \beta} f(t, x(t))\right| \\
& \leqslant \frac{w(a)}{w(t)}|x(a)|+\mathcal{J}_{a, w}^{\alpha, \beta}|f(t, x(t))| .
\end{aligned}
$$


Since $w(a) \leqslant w(t)$ for all $t \geqslant a, f$ is Lipchitz continuous and $t \leqslant b$, we deduce that $|f(t, x(t))|$ is bounded by a constant $m$ and

$$
\begin{aligned}
|(\Upsilon x)(t)| & \leqslant|x(a)|+m^{J} \mathcal{J}_{a, w}^{\alpha, \beta}(1) \\
& \leqslant|x(a)|+m\left(\frac{1-\alpha}{N(\alpha)}+\frac{\alpha(b-a)^{\beta}}{N(\alpha) \Gamma(\beta+1)}\right)
\end{aligned}
$$

which implies that the Picard's operator is well defined. Moreover, for all $x, y \in \mathcal{C}$ and $t \in[a, b]$, we have

$$
\begin{aligned}
\left|\left(\gamma_{x}\right)(t)-\left(\Upsilon_{y}\right)(t)\right|= & \left|\mathcal{J}_{a, w}^{\alpha, \beta} f(t, x(t))-\mathcal{J}_{a, w}^{\alpha, \beta} f(t, y(t))\right| \\
= & \mid \frac{1-\alpha}{N(\alpha)}(f(t, x(t))-f(t, y(t))) \\
& +\frac{\alpha}{N(\alpha)} R_{\mathcal{J}}^{\beta} \beta, w(f(t, x(t))-f(t, y(t))) \mid \\
\leqslant & \frac{1-\alpha}{N(\alpha)}|f(t, x(t))-f(t, y(t))| \\
& \left.+\frac{\alpha}{N(\alpha)} R L_{\mathcal{J}}^{\beta}{ }_{a, w} \mid f(t, x(t))-f(t, y(t))\right) \mid \\
\leqslant & \frac{1-\alpha}{N(\alpha)} L|x-y|+\frac{\alpha}{N(\alpha)} L\|x-y\|_{e} \frac{(t-a)^{\beta}}{\Gamma(\beta+1)} \\
\leqslant & L\left(\frac{1-\alpha}{N(\alpha)}+\frac{\alpha(b-a)^{\beta}}{N(\alpha) \Gamma(\beta+1)}\right)\|x-y\|_{e} .
\end{aligned}
$$

Hence,

$$
\left\|\Upsilon_{x}-r_{y}\right\|_{e} \leqslant L\left(\frac{1-\alpha}{N(\alpha)}+\frac{\alpha(b-a)^{\beta}}{N(\alpha) \Gamma(\beta+1)}\right)\|x-y\|_{e}
$$

Since $L\left(\frac{1-\alpha}{N(\alpha)}+\frac{\alpha(b-a)^{\beta}}{N(\alpha) \Gamma(\beta+1)}\right)<1$, we deduce that $\Upsilon$ is a contraction mapping. Therefore, by applying the Banach contraction mapping principle, problem (4.1) admits a unique solution.

To illustrate the result presented in the above theorem, we propose the following examples.

Example 4.1. We consider the following Cauchy problem

$$
\left\{\begin{array}{l}
\mathcal{D}_{0, w}^{\alpha, \frac{1}{2}} x(t)=\frac{1}{2} \mathrm{t}^{2} \chi(t), \quad \mathrm{t} \in[0,1], \quad \mathrm{N}(\alpha)=1, \\
x(0)=1 .
\end{array}\right.
$$

Here, $f(t, x(t))=\frac{1}{2} t^{2} \chi(t)$ and it satisfies

$$
|f(t, x)-f(t, y)|=\frac{1}{2} t^{2}|x-y| \leqslant \frac{1}{2}|x-y|
$$

Hence, the condition $\left(\mathcal{A}_{1}\right)$ holds with $\mathrm{L}=\frac{1}{2}$. Since $\Gamma(\beta+1)=\Gamma\left(\frac{3}{2}\right)=\frac{\sqrt{\pi}}{2}$, we have

$$
\mathrm{L}\left(\frac{1-\alpha}{\mathrm{N}(\alpha)}+\frac{\alpha(\mathrm{b}-\mathrm{a})^{\beta}}{\mathrm{N}(\alpha) \Gamma(\beta+1)}\right)=\frac{1}{2}\left(1-\frac{(\sqrt{\pi}-2) \alpha}{\sqrt{\pi}}\right) \leqslant \frac{1}{2}<1 .
$$

It follows from Theorem 4.3 that system (4.4) has a unique solution.

Example 4.2. Consider the following Cauchy problem

$$
\left\{\begin{array}{l}
\mathcal{D}_{0, w}^{\alpha, 2} x(t)=\frac{e^{-2 t}}{3} \frac{|x(t)|}{1+|x(t)|}, \quad t \in[0,1], \quad N(\alpha)=1 \\
x(0)=0 .
\end{array}\right.
$$


In this case, $\beta=2$ and $f(t, x(t))=\frac{e^{-2 t}}{3} \frac{|x(t)|}{1+|x(t)|}$. For all $t \in[0,1]$ and $x, y \in \mathbb{R}$, we have

$$
|f(t, x)-f(t, y)| \leqslant \frac{e^{-2 t}}{3}|| x|-| y|| \leqslant \frac{1}{3}|x-y| .
$$

Then the condition $\left(\mathcal{A}_{1}\right)$ holds with $\mathrm{L}=\frac{1}{3}$. Furthermore,

$$
\mathrm{L}\left(\frac{1-\alpha}{\mathrm{N}(\alpha)}+\frac{\alpha(\mathrm{b}-\mathrm{a})^{\beta}}{\mathrm{N}(\alpha) \Gamma(\beta+1)}\right)=\frac{1}{3}\left(1-\frac{\alpha}{2}\right) \leqslant \frac{1}{3}<1
$$

Since all assumptions of Theorem 4.3 are satisfied, then the system $(4.5)$ has a unique solution on $[0,1]$.

\section{Application}

In this section, we apply the results obtained in the previous sections in order to establish the conditions for Ulam-Hyers stability of system (4.1). First, we recall the definition of this type of stability. Let us consider the following inequality

$$
\left|\mathcal{D}_{a, w}^{\alpha, \beta} x(t)-f(t, x(t))\right| \leqslant \epsilon .
$$

Definition 5.1. System (4.1) is Ulam-Hyers stable if there exists a real number $L_{f}>0$ such that for each $\epsilon>0$ and for each solution $y \in \mathcal{C}$ of inequality (5.1), there exists a solution $x \in \mathcal{C}$ of (4.1) with

$$
|y(t)-x(t)| \leqslant \epsilon L_{f}, \quad t \in[a, b] .
$$

Theorem 5.2. Assume that the assumptions of Theorems 4.2 and 4.3 are satisfied. Then (4.1) is Ulam-Hyers stable.

Proof. Let $\epsilon>0$ and $y \in \mathcal{C}$ be a function which satisfies inequality (5.1). Then there exists a function $g(t)$ verifies $|g(t)| \leqslant \epsilon$ such that

$$
\mathcal{D}_{a, w}^{\alpha, \beta} y(t)-f(t, y(t))=g(t)
$$

From (2.4), we obtain

$$
y(t)-\frac{w(a) y(a)}{w(t)}-\mathcal{J}_{a, w}^{\alpha, \beta} f(t, y(t))=\mathcal{J}_{a, w}^{\alpha, \beta} g(t)
$$

Hence,

$$
\begin{aligned}
\left|y(t)-\frac{w(a) y(a)}{w(t)}-\mathcal{J}_{a, w}^{\alpha, \beta} f(t, y(t))\right| & =\left|\mathcal{J}_{a, w}^{\alpha, \beta} g(t)\right| \\
& \leqslant \frac{1-\alpha}{N(\alpha)}|g(t)|+\frac{\alpha}{N(\alpha)}\left|{ }^{R L} \mathcal{J}_{a, w}^{\beta} g(t)\right| \\
& \leqslant \frac{1-\alpha}{N(\alpha)} \epsilon+\frac{\alpha}{N(\alpha)} \frac{\epsilon}{\Gamma(\beta+1)}(t-a)^{\beta} \\
& \leqslant \epsilon\left(\frac{1-\alpha}{N(\alpha)}+\frac{\alpha}{N(\alpha)} \frac{(b-a)^{\beta}}{\Gamma(\beta+1)}\right) .
\end{aligned}
$$

Let $x(t)$ be the unique solution of (4.1) with $x(a)=y(a)$. Then

$$
x(t)=\frac{w(a) y(a)}{w(t)}+\mathcal{J}_{a, w}^{\alpha, \beta} f(t, x(t)) .
$$

Now, we have

$$
|y(t)-x(t)|=\left|y(t)-\frac{w(a) y(a)}{w(t)}-\mathcal{J}_{a, w}^{\alpha, \beta} f(t, y(t))+\mathcal{J}_{a, w}^{\alpha, \beta}(f(t, y(t))-f(t, x(t)))\right|
$$




$$
\begin{aligned}
& \leqslant\left|y(t)-\frac{w(a) y(a)}{w(t)}-\mathcal{J}_{a, w}^{\alpha, \beta} f(t, y(t))\right|+\mathcal{J}_{a, w}^{\alpha, \beta}|f(t, y(t))-f(t, x(t))| \\
& \leqslant \epsilon\left(\frac{1-\alpha}{N(\alpha)}+\frac{\alpha}{N(\alpha)} \frac{(b-a)^{\beta}}{\Gamma(\beta+1)}\right)+\mathcal{J}_{a, w}^{\alpha, \beta}|f(t, y(t))-f(t, x(t))| .
\end{aligned}
$$

Since f satisfies the Lipschitz condition given in Theorem 4.3, we get

$$
\left.|y(t)-x(t)| \leqslant \epsilon\left(\frac{1-\alpha}{N(\alpha)}+\frac{\alpha}{N(\alpha)} \frac{(b-a)^{\beta}}{\Gamma(\beta+1)}\right)+L_{\mathcal{J}}^{\alpha, \beta}, \mid y(t)\right)-x(t) \mid .
$$

Applying the Gronwall inequality in Corollary 3.5 to the above inequality, we get

$$
\begin{aligned}
|y(t)-x(t)| & \leqslant \frac{\epsilon\left[(1-\alpha) \Gamma(\beta+1)+\alpha(b-a)^{\beta}\right]}{\Gamma(\beta+1)[N(\alpha)-(1-\alpha) L]} E_{\beta}\left(\frac{\alpha L(t-a)^{\beta}}{N(\alpha)-(1-\alpha) L}\right) \\
& \leqslant \frac{\epsilon\left[(1-\alpha) \Gamma(\beta+1)+\alpha(b-a)^{\beta}\right]}{\Gamma(\beta+1)[N(\alpha)-(1-\alpha) L]} E_{\beta}\left(\frac{\alpha L(b-a)^{\beta}}{N(\alpha)-(1-\alpha) L}\right) .
\end{aligned}
$$

Thus,

$$
|y(t)-x(t)| \leqslant \epsilon L_{f},
$$

where $\mathrm{L}_{\mathrm{f}}=\frac{(1-\alpha) \Gamma(\beta+1)+\alpha(\mathrm{b}-\mathrm{a})^{\beta}}{\Gamma(\beta+1)[\mathrm{N}(\alpha)-(1-\alpha) \mathrm{L}]} \mathrm{E}_{\beta}\left(\frac{\alpha \mathrm{L}(\mathrm{b}-\mathrm{a})^{\beta}}{\mathrm{N}(\alpha)-(1-\alpha) \mathrm{L}}\right)$. Therefore, (4.1) is Ulam-Hyers stable.

Remark 5.3. Theorem 5.2 extends the result of the Ulam-Hyers stability stability presented in [19, Theorem 4.1] for the GHF derivative when $w(t)=1$ and $\beta=\alpha$.

\section{Conclusion}

The cauchy problem formulated by (4.1) can better describe the dynamics of many biological systems arising from epidemiology, virology and viral immunology in order to take into account the memory effect. In this work, we have proved the existence and uniqueness of solutions of (4.1). By means of the obtained Gronwall inequality and based on the conditions for the existence and uniqueness of solutions, we have established the stability of (4.1) in the sense of Ulam-Hyers.

The main results obtained in this study are based on the GHF derivative defined by (2.1) in the case when $\gamma=\beta$. It will be interesting to study the general case when $\gamma \neq \beta$. This will be done in our future work.

\section{Acknowledgment}

The authors would like to thank the editor and anonymous reviewers for their constructive comments and suggestions, which have improved the quality of the manuscript.

\section{References}

[1] Y. Adjabi, F. Jarad, T. Abdeljawad, On generalized fractional operators and a Gronwall type inequality with applications, Filomat, 31 (2017), 5457-5473. 1, 3, 3.4

[2] O. P. Agrawal, Some generalized fractional calculus operators and their applications in integral equations, Fract. Calc. Appl. Anal., 15 (2012), 700-711. 1

[3] A. Akgül, Y. Khan, A novel simulation methodology of fractional order nuclear science model, Math. Methods Appl. Sci., 40 (2017), 6208-6219. 1

[4] M. Al-Refai, On weighted Atangana-Baleanu fractional operators, Adv. Difference Equ., 2020 (2020), 11 pages. 1, 2

[5] M. Al-Refai, A. M. Jarrah, Fundamental results on weighted Caputo-Fabrizio fractional derivative, Chaos Solitons Fractals, 126 (2019), 7-11. 1 
[6] J. Alzabut, T. Abdeljawad, F. Jarad, W. Sudsutad, A Gronwall inequality via the generalized proportional fractional derivative with applications, J. Inequal. Appl., 2019 (2019), 12 pages. 1, 3.4

[7] A. Atangana, D. Baleanu, New fractional derivatives with nonlocal and non-singular kernel: Theory and application to heat transfer model, Thermal Sci., 20 (2016), 763-769. 1, 2, 2.3

[8] D. Baleanu, A. Fernandez, On some new properties of fractional derivatives with Mittag-Leffler kernel, Commun. Nonlinear Sci. Numer. Simul., 59 (2018), 444-462. 2

[9] M. A. Bayrak, A. Demir, E. Ozbilge, On solution of fractional partial differential equation by the weighted fractional operator, Alexandria Eng. J., 59 (2020), 4805-4819. 1

[10] M. Caputo, M. Fabrizio, A new definition of fractional derivative without singular kernel, Progr. Differ. Appl., 1 (2015), 73-85. 1, 2

[11] K. R. Cheneke, K. Purnachandra Rao, G. Kenassa Edessa, Application of a new generalized fractional derivative and rank of control measures on cholera transmission dynamics, Int. J. Math. Math. Sci., 2021 (2021), 9 pages. 1

[12] J. Dixon, S. McKee, Weakly singular discrete Gronwall inequalities, Z. Angew. Math. Mech., 66 (1986), 535-544. 1

[13] J. D. Djida, A. Atangana, I. Area, Numerical computation of a fractional derivative with non-local and non-singular kernel, Math. Model. Nat. Phenom., 12 (2017), 4-13. 2

[14] N. Faraz, Y. Khan, A. Anjum, Mathematical modelling of unsteady fractional Phan Thien Tanner fluid, Alexandria Eng. J., 59 (2020), 4391-4395. 1

[15] M. Fardi, Y. Khan, A novel finite difference-spectral method for fractal mobile/immobiletransport model based on CaputoFabrizio derivative, Chaos Solitons Fractals, 143 (2021), 8 pages. 1

[16] T. H. Gronwall, Note on the derivatives with respect to a parameter of the solutions of a system of differential equations, Ann. of Math. (2), 20 (1919), 292-296. 1

[17] K. Hattaf, A new generalized definition of fractional derivative with non-singular kernel, Computation, 8 (2020), 9 pages. $1,2.1,2,2.2$

[18] K. Hattaf, On some properties of the new generalized fractional derivative with non-singular kernel, Math. Probl. Eng., 2021 (2021), 6 pages. 2.4

[19] F. Jarad, T. Abdeljawad, Z. Hammouch, On a class of ordinary differential equations in the frame of Atangana-Baleanu fractional derivative, Chaos Solitons Fractals, 117 (2018), 16-20. 1, 3.4, 5.3

[20] Y. Khan, M. A. Khan, Fatmawati, N. Faraz, A fractional Bank competition model in Caputo-Fabrizio derivative through Newton polynomial approach, Alexandria Eng. J., 60 (2021), 711-718. 1

[21] N. Sene, Stability analysis of the generalized fractional differential equations with and without exogenous inputs, J. Nonlinear Sci. Appl., 12 (2019), 562-572. 1

[22] N. Sene, Second-grade fluid model with Caputo-Liouville generalized fractional derivative, Chaos Solitons Fractals, 133 (2020), 7 pages. 4

[23] N. Sene, Theory and applications of new fractional-order chaotic system under Caputo operator, Int. J. Optim. Control Theor. Appl., 12 (2022), 20-38. 1 